\title{
Coulisses
}

Revue de théâtre

15 | Hiver 1997

Varia

\section{La Cerisaie d'Anton Tchekhov}

Conférence du Jeudi 12 décembre 1996 dans le cadre du « Regard du Spectateur » organisé par l'Université Ouverte

\section{Serge Rolet}

\section{(2) OpenEdition}

1 Journals

Édition électronique

URL : http://journals.openedition.org/coulisses/5127

DOI : $10.4000 /$ coulisses. 5127

ISSN : 2546-9460

Éditeur

Presses universitaires de Franche-Comté

\section{Édition imprimée}

Date de publication : 1 janvier 1997

Pagination : 52-60

ISSN : 1150-594X

\section{Référence électronique}

Serge Rolet, «La Cerisaie d'Anton Tchekhov », Coulisses [En ligne], 15 | Hiver 1997, mis en ligne le 26 avril 2019, consulté le 22 octobre 2019. URL : http://journals.openedition.org/coulisses/5127 ; DOI $10.4000 /$ coulisses.5127

Ce document a été généré automatiquement le 22 octobre 2019.

Coulisses 


\section{La Cerisaie d'Anton Tchekhov}

Conférence du Jeudi 12 décembre 1996 dans le cadre du « Regard du Spectateur » organisé par l'Université Ouverte

\section{Serge Rolet}

1 La pièce est créée le 17 janvier 1904, jour anniversaire de l'auteur, et en sa présence. Pendant qu'il écrit La Cerisaie, à Yalta, Tchékhov est très malade. Il travaille lentement, avec difficulté; certains jours il n'écrit pas plus de dix lignes. Dans ses lettres, sa femme, l'actrice Olga Knipper, qui doit jouer Ranevskaïa, le

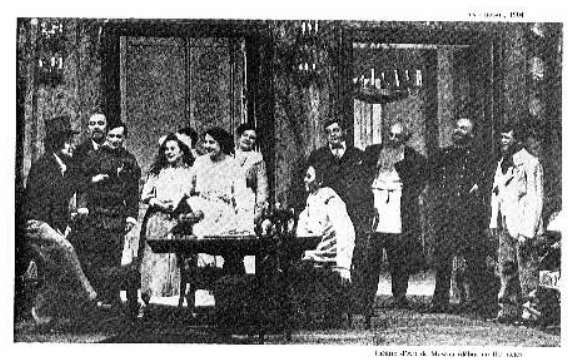
presse de finir : elle craint que la pièce ne voie jamais le jour. Le texte une fois achevé, c'est pour Tchékhov un soulagement plus qu'une satisfaction. Il a l'impression de se répéter. ${ }^{1}$

\section{La Cerisaie, une action sans surprise ${ }^{2}$}

2 De ce que raconte la pièce, on trouve la trace depuis longtemps chez Tchékhov. L'histoire de la vente de la propriété familiale est partout dans ses récits de jeunesse, dans ses pièces. Le récit Fleurs tardives, qui date du début des années 1880, montre un Allemand, Boris Peltzer, qui acquiert pour trois fois rien le domaine d'une noble personne à laquelle il avait promis de prêter de l'argent. Nous sommes tout près de $L a$ Cerisaie.

Le sujet est aussi devenu un classique de la littérature russe, ne serait-ce que parce que depuis l'abolition du servage (en 1861) les ventes aux enchères de grandes propriétés se multiplient. Les journaux sont remplis d'annonces de ventes: les nobles ruinés sont remplacés par les nouveaux riches, comme Lopakhine. Tout cela est connu. Leïkine, l'un des premiers patrons de presse auxquels Tchékhov ait eu affaire, avait acheté en 1885 le domaine des comtes Stroganov. Celui des princes Golitsyne avait été loti. Les 
propriétaires de la villa, où la famille de Tchékhov passe plusieurs étés au milieu des années 1880 , échafaudaient des plans en fonction du passage d'une future voie ferrée à proximité de leurs terres. On le voit bien, la valeur de la pièce n'est pas là, dans la trame narrative, mais dans l'atmosphère, bien entendu, la fameuse ambiance tchékhovienne, le lyrisme retenu, la nostalgie.

L'événement qui décide de l'avenir du domaine et du sort des personnages de $\mathbf{L a}$ Cerisaie a lieu en dehors de la scène. Sous les yeux des spectateurs ne se déroule pour ainsi dire aucune action. Les gens ne font qu'attendre; ils sont tous (sauf Firs) entre deux trains, deux villes. Lopakhine regarde tout le temps sa montre, Ranevskaïa n'a que Paris en tête, tout comme lâcha, le laquais, etc. Toute la pièce n'est qu'une grande pause entre le passé et l'avenir. Cette pause est remplie par quelques paroles, mais surtout par une douleur difficile à définir. Les acteurs n'ont pas de jeu à jouer. ${ }^{3}$

Dans La Cerisaie, il n'y a pas d'oppositions de personnages, ni de coups de pistolet comme dans Oncle Vania, de duels mortels, comme dans Les trois sœurs, d'amours non partagées, comme dans La Mouette. Il n'y a rien, sinon les rapports troubles de Lopakhine et de Ranevskaïa - vieil attachement de l'ancien esclave pour ses maîtres, ou volonté de posséder. Leur antagonisme larvé se défait dans la grande indulgence qui, presque toujours, adoucit les relations entre les êtres. Sur la scène, c'est le quotidien qui est au premier plan. Prendre le café (ou autre chose: "Tu m'apporteras du kvass.»), s'asseoir, se lever, rêvasser, dire des bêtises ("Bille en tête, avec effet à droite.»), être incommodé par l'odeur des laquais ("Tu sens la basse-cour!»), s'attendrir sur les cerisiers en fleurs : voilà de quoi la vie est faite.

Pourquoi préférer le quotidien? Quel sens a cette option, nouvelle dans le théâtre russe? Quel lien existe-t-il entre les gestes les plus ordinaires des personnages et l'atmosphère tchékhovienne, la toska? Cette "maladie » russe vient, justement, du quotidien, des objets environnants, du cadre, des gens qu'on côtoie : elle vient des sensations. Là est toute la dramaturgie tchékhovienne. Les émotions des personnages sont causées par l'antique armoire, par les cerisiers, par l'apparition du précepteur de Gricha, l'enfant mort noyé.

En tant que médecin, ${ }^{4}$ Tchékhov savait à quel point le monde intérieur de l'homme est lié au milieu, au quotidien. Il construit un univers dont le quotidien est la base. Simplement le quotidien «étouffe l'esprit ». "Les gens, écrit Tchékhov, prennent leur repas, ils sont à table, et voici que leur destin prend forme, que leur vie se brise.» Tchékhov ne décrit pas les états d'âme ; chez lui la souffrance, la douleur, la passion répugne à se montrer au grand jour, elle ne donne lieu le plus souvent qu'au silence. D'ordinaire Tchékhov ne mentionne que la cause objective, extérieure, appartenant à l'univers du quotidien, le motif qui occasionne l'état d'âme. Il se refuse à l'analyse psychologique, dont la prose narrative et le théatre de son temps sont si riches. C'est pourquoi ce que voit le personnage, ce qu'il entend (les sensations auditives, on l'a souvent relevé, sont particulièrement importantes chez Tchékhov) est à ce point souligné. Les images de la réalité, les impressions venues du monde environnant, ainsi, ne décrivent ni ne reflètent : elles causent la souffrance. ${ }^{5}$

\section{La Cerisaie et le non-dit}

6 Au passage, je voudrais dire deux mots de la mort du petit garçon de Ranevskaïa. On peut voir dans cet accident l'origine de tous les maux de l'héroïne (sans lui, pas de 
chagrin, pas de voyage à Paris, pas de ruine, pas de vente du domaine). Cette interprétation s'oppose à celle que je viens d'esquisser : ce n'est pas le quotidien, les sensations immédiates qui priment, c'est un événement, la noyade. Mais la noyade ellemême, comment la comprendre? Ce n'est pas un signe de Dieu, je ne sais quelle punition pour quelque faute commise, ainsi que le croit Ranevskaïa. C'est beaucoup plus simple. Chez Tchékhov, d'une manière générale, Dieu est absent, sinon dans les propos naïfs des vieux serviteurs. Dans ses lettres, il n'en est pas question. A l'égard de la foi, de la dévotion, la position de Tchékhov est gentiment ironique. A sa mère, qui était très pieuse, il demandait avec malice : «Dites, chère mère, les moines portent-ils des caleçons?» Dostoïevski se disait enfant de la foi et de l'incroyance; pour lui, comme d'ailleurs pour Tolstoï, toute morale avait sa source dans la religion. Pour sa part, Tchékhov note, ironique : «Quand on a soif, on se dit qu'on va boire la mer tout entière : c'est la foi ; mais quand on commence à boire, on s'aperçoit qu'on va s'arrêter après un ou deux verres; ça, c'est la science ». Dans la perspective de Tchékhov, celle, une fois encore, de la pratique médicale, de la science, il faut, je crois, considérer la mort du petit Gricha comme un pur et simple hasard. Tel est toujours à ses yeux le cas de la mort : elle est un hasard. Dans Les groseilliers, on trouve l'idée que « tôt ou tard la vie montre ses griffes». La maladie arrive par hasard. Dans la vision qu'en ont les simples gens, dans la vie quotidienne, elle n'a pas de cause. Mais la réflexion du médecin est scientifique justement parce qu'elle se développe et se perfectionne par l'accumulation de hasards répétés; leur multiplicité statistique débouche sur la généralisation, elle donne l'expérience, le savoir systématique. On a l'impression que chez Tchékhov, la structure de la narration, en surface au moins, est fragmentaire, sans unité. L'unité est fournie par la méthode, par la réflexion.

Revenons à la souffrance, au lien à première vue si étrange qui la relie aux êtres environnants. C'est la venue de Trofimov qui « cause » la douleur de Ranevskaïa; c'est la vue des cerisiers qui « cause » la tristesse, la nostalgie des personnages. Le verger est donc tout autre chose qu'un élément du décor; il est inséparable de l'état d'âme. Les cerisiers, c'est l'image embellie du passé, de l'enfance, du bonheur, peut-être. Entre les arbres et l'armoire dont il tombe une poussette, et qui a été assemblée cent ans plus tôt, il y a une correspondance symbolique évidente. Le moyen terme entre ces deux éléments matériels, c'est le lieu de l'action, cette ancienne chambre d'enfants, où est restée l'armoire, et dont les fenêtres donnent sur la cerisaie.

7 Chez Tchékhov, l'émotion est contenue, discrète ; elle ne donne pas lieu à déclaration. Pas d'épanchement: l'émotion est perceptible pour le spectateur, bien qu'elle soit le plus souvent sans paroles, ou, au moins, que les mots soient sans rapport avec la douleur qui s'exprime comme à travers eux. Ce sont les arbres en fleurs, l'armoire, le vieux valet qui causent la douleur : cela, le spectateur le sent. On peut bien entendu se contenter de décrire cette découverte dramaturgique de Tchékhov: que les émotions naissent directement des sensations, du contact avec le milieu, mais on peut aussi chercher à comprendre dans quelle démarche d'ensemble s'inscrit ce choix de la nonparole. C'est cette réticence à l'égard du langage, ou, au moins, du discours, que je voudrais maintenant évoquer. On pourra à cette occasion cerner d'un peu plus près ce que Tchékhov appelle la maladie russe: cette maladie est en effet avant tout une maladie de la parole. 


\section{Tchékhov ou le refus de tout système}

8 Il faut tout d'abord lever une ambiguïté au sujet de l'abstention de Tchékhov à prescrire d'éventuels remèdes capables de guérir les russes de la maladie dont il s'agit. Quand on évoque le "silence " de Tchékhov, on parle de son refus d'énoncer des solutions aux grands problèmes de son temps. ${ }^{6}$ De là à l'idée que Tchékhov ne voit pas de solution, il y a un pas que, je crois, il faut bien se garder de franchir. Que la maladie russe soit incurable, c'est Gaïev qui le dit dans La Cerisaie («Si pour guérir une maladie on vous propose des tas de remèdes, c'est que la maladie est incurable »). Tchékhov n'a pas de doute quant à ce qu'il faut faire. Il déploie même dans l'accomplissement de la tâche qu'il s'assigne une très grande énergie, une étonnante force morale ${ }^{7}$. Simplement, la difficulté, le caprice de la maladie, c'est que les solutions ne peuvent pas être prescrites dans la littérature ou sur la scène.

\section{La Cerisaie}

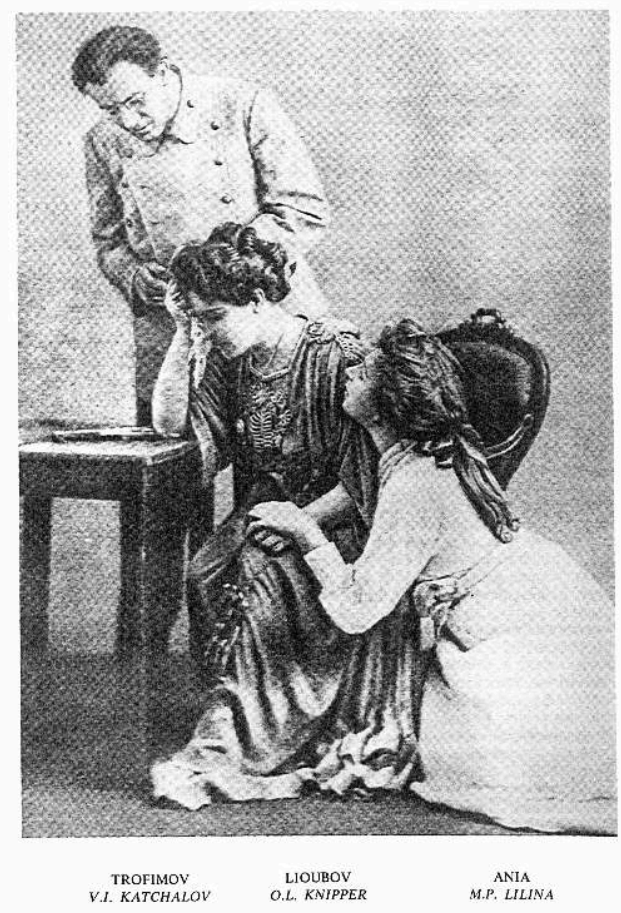

Photo Théâtre d'Art de Moscou

Les lettres que Tchékhov écrit à ses frères permettent de se faire une idée de ce qu'il voulait dire. Alexandre et Nicolas Tchékhov avaient du talent; ils n'étaient pas dépravés ${ }^{8}$. Leur défaut, c'était d'être des rêveurs, des naïfs, d'attendre de la vie plus qu'ils ne pouvaient en recevoir. Alexandre et Nicolas étaient proches des « humiliés et offensés » dont parle Dostoïevski. Ce qui a brisé Nicolas Tchékhov, ce n'est pas des travaux trop durs (il travaillait très peu), quelque grand-œuvre au-dessus de ses forces, ni une passion destructrice ou sublime, mais l'habituel penchant des russes pour le train-train indolore, les amours hasardeuses, les grands projets jamais poussés plus loin que le rêve, abandonnés avant que l'inspiration ne vienne. L'échec, les vies gâchées, tout cela vient, pour Tchékhov, de l'attendrissement sur soi-même, d'un manque de 
simplicité. Il écrit à l'un de ses frères: "Tu te plains à moi qu' "on ne te comprend pas!!". De cela, même Gœthe et Newton ne se plaignaient pas... Seul le Christ s'en plaignait, encore ne parlait-il pas de son "moi", mais de son enseignement... Toi, on te comprend parfaitement ». Ce qu'il faut, c'est de la netteté, de la tenue avec ceux qui vous sont inférieurs socialement, du respect pour autrui, de la constance au travail. Il faut exclure la violence, la tyrannie, de notre comportement personnel. « Une situation difficile, dit Tchékhov, le caractère pénible des femmes avec lesquelles il te faut vivre, la bêtise des cuisinières, un travail de forçat, une vie d'anathème, et tout le reste, ne peuvent servir à justifier ton despotisme. Mieux vaut être victime que bourreau ». Les frères de Tchékhov tiraient des plans sur la comète, remettaient leurs obligations à plus tard, jouaient leur argent à la loterie, et noyaient leur chagrin dans l'alcool. Et alors commençait un discours typiquement russe : la jérémiade, les longues plaintes contre la dureté de la vie, contre le milieu, qui vous a volé votre jeunesse, et ainsi de suite. «Celui qui pense être coupable s'invente des justifications extérieures : l'ivrogne prend prétexte de son chagrin ». Il faut ne pas avoir à se sentir coupable, se garder comme de la peste des «bonnes raisons": voilà donc bien la solution aux problèmes que Tchékhov se limite à poser correctement. Il faut œuvrer au bien commun, agir, au lieu de continuer à parler comme le font les polémistes, les publicistes, les écrivains, libéraux ou conservateurs, peu importe. Comment? Ouvrir des écoles, soigner les moujiks pour rien, respecter les petits (les enfants, les domestiques).

Tchékhov a fait ouvrir trois écoles modèles, dont il a payé la construction de sa poche. Il voyait entre mille et trois mille malades par an, sans jamais demander d'honoraires : les gens ne pouvaient pas le payer. Toute sa vie, il a été à court d'argent, non parce qu'il vivait comme un prince, mais parce qu'il consacrait beaucoup de ce qu'il gagnait à des œuvres sociales. Son train de vie était loin de celui de ses amis et patrons Leïkine ou Souvorine. Jamais il n'a porté un manteau de plus de cinquante roubles (une misère).

Tchékhov ne recherchait pas les honneurs, c'est le moins qu'on puisse dire. Son activité de médecin et de pionnier dans l'instruction du peuple avaient été récompensées par le tsar: un décret lui conférait la noblesse héréditaire et le décorait de l'ordre de Stanislas. Tchékhov a ignoré ces dispositions, dont, semble-t-il, nul n'a rien su jusqu'à ce qu'en 1930, un chercheur découvre le précieux papier. Il n'a jamais porté la décoration, ni ne s'est prévalu de son titre. De même, il a décliné l'invitation que lui lançait la femme du grand-prince Sergueï Alexandrovitch, frère du tsar. "Dis-lui bien, demande-t-il à sa femme, chargée de transmettre l'invitation, que je ne puis aller chez elle, et que jamais elle ne me verra». Tchékhov avait en horreur les grands de ce monde, les grands sages, Diogène, Tolstoï, car il distinguait dans leur attitude la violence que produit toute position dominante. La domination acquise par la parole ne fait pour lui pas exception.

11 Si Tchékhov ne donne pas, explicitement, sa position, les solutions qu'il propose, ce n'est donc pas par absence d'idées, par indifférence, par esthétisme, mais parce que la parole en Russie est depuis trop longtemps paralysante, qu'elle est un prétexte à l'inaction. Pour Tchékhov, énoncer des solutions, c'est le meilleur moyen de garder bonne conscience tout en restant les bras croisés. Parler, éclairer le public, tel est le vice des intellectuels, des universitaires, des médecins, des avocats, etc. "Je n'ai pas confiance en notre intelligentsia, hypocrite, fausse, hystérique, mal dégrossie, paresseuse, je me méfie d'elle même quand elle souffre et se plaint, car ses oppresseurs sont sortis de ses propres flancs » (dans une lettre, fameuse, à I. Orlov, de 1899). 


\section{Tchékhov et l'engagement littéraire} Polonski, Plechtchéïev) veulent tirer Tchékhov de la voie où il s'est malencontreusement (selon eux) engagé : ils veulent le mettre sur la voie romanesque. Tchékhov répond doucement qu'il ne pourra pas sortir « comme ça » de l'ornière où il est engagé, celle de la petite presse. Mais ce n'est pas l'habitude qui l'empêche d'écrire un roman. Le cœur du problème, c'est une question de vision du monde. C'est au nom du bien commun, de la paix civile, du respect d'autrui, qu'il rejette le roman, genre à ses yeux trop discursif, philosophique, didactique, cérébral. Les efforts de Grigorovitch, Polonski, Plechtchéiev ne donnent aucun résultat : Tchékhov essaie d'écrire un roman, mais il n'y parvient pas. Cette forme est décidément trop étrangère à son attitude.

Il n'a pas non plus écrit de préfaces, de postfaces, d'articles, ni de commentaires, comme on en trouve à profusion chez les grands auteurs de son temps (Tolstoï et Dostoïevski, par exemple). Il y a chez lui une grande réticence envers la parole, l'affirmation de quelque vérité que ce soit. Il dit dans une lettre à Souvorine de 1888 qu'« un grand progrès serait accompli si ceux qui parlent au peuple (les écrivains, S. R.) avouaient tout simplement qu'ils ne comprennent rien à rien ».

Quels sont donc les vices du discours? La vacuité, l'inutilité, et, par-dessus tout, la haine.

Il faut revenir sur l'opposition que j'ai établie entre la "grande littérature » et la « petite presse ». ${ }^{9}$ Tchékhov, longtemps, écrit dans la petite presse parce qu'elle seule permet de donner libre cours à l'humour, au rire qui libère de l'oppression, évacue le sérieux des intellectuels qui parlent haut mais ignorent tout de la vie; les petits textes humoristiques mettent en scène la vie, la vraie, celle du quotidien, des situations prosaïques réelles. L'humoresque a une valeur sociologique précieuse. Grâce à tous ces petits textes, à tous les récits de Tchékhov, on compte dans l'ensemble de son œuvre près de huit mille personnages (chez Balzac, trois mille seulement) : nous avons là une encyclopédie de la vie russe. Mais dans cette petite presse, dans ses relations avec son patron, Leïkine, le propriétaire des Eclats, Tchékhov se sent dès l'origine dans un monde étranger.

Ce n'est pas seulement le style, la hâte avec laquelle il faut remettre sa copie qui le gênent, c'est la vision du monde, de ce que doit être un texte. La manière de poser les problèmes n'est pas la même.

16 La différence de vision entre Tchékhov et Leïkine est la même que celle qui le sépare, plus tard, de Souvorine. Leïkine ne comprend pas les œuvres « sérieuses » de Tchékhov, celles qui dépassent la longueur de quelques dizaines de lignes. Par exemple, il n'arrive pas à finir La steppe. "Voilà ce que je pense, écrit-il à Tchékhov, dans vos petits textes, là où vous apparaissez comme humoriste, vous êtes un grand maitre ». Autrement dit, pour Leïkine, le vrai Tchékhov est dans L'Ours plutôt que dans La Cerisaie! Ce que demande Leïkine, ce sont des pièces humoristiques de quelques lignes. Il écrit à Tchékhov (1884):

Vous me faites le reproche d'orienter Oskolki (Les éclats) vers le billet satirique. Je m'étonne de ce grief. Si je le pouvais, je mettrais dans Les éclats des billets satiriques de la première à la dernière ligne. Seulement je ne trouve personne (pour les écrire, S. R.). 
Et il ajoute, un peu plus loin: «Non, vous n'êtes pas un journaliste ». Tchékhov tend vers des formes plus longues, plus graves, que le billet satirique. En 1884 encore, il fait part à Leïkine de ses réflexions : « Je lis Les éclats avec attention. C'est un bon journal, le meilleur en tout cas de tous les journaux humoristiques... Mais ne vous semble-t-il pas que Les éclats sont un peu secs? Ce qui leur donne cette sécheresse, à mon avis, c'est la multiplicité des billets satiriques. »

En 1886, année de la première critique qui lui est consacrée, Tchékhov est encore très peu connu. I. Bounine dit que les gens qui avaient de idées s'intéressaient peu à lui. Certains même refusaient de le lire en raison des pseudonymes "vulgaires » qu'il prenait, et que « jamais Tourgueniev ni Tolstoï n'auraient pris».

Mais même parmi les gens qui lisaient la petite presse (les boutiquiers, les artisans, le peuple des grandes villes, le public des "boulevards", comme on dirait en France à cette époque, ne le connaissent pas non plus. Après La Mouette, Tchékhov raconte dans une lettre (mars 1895) :

Ma mère va chez notre boucher, elle lui réclame un bon morceau, car, dit-elle, nous recevions Leïkine, de Pétersbourg. "Celui qui écrit des livres?» s'étonne le boucher, et il choisit une viande excellente. Cela veut dire que notre boucher ne sait pas que moi aussi, j'écris des livres, puisque dans ce qu'il me donne, il n'y a que des nerfs.

\section{La Cerisaie (acte IV)}
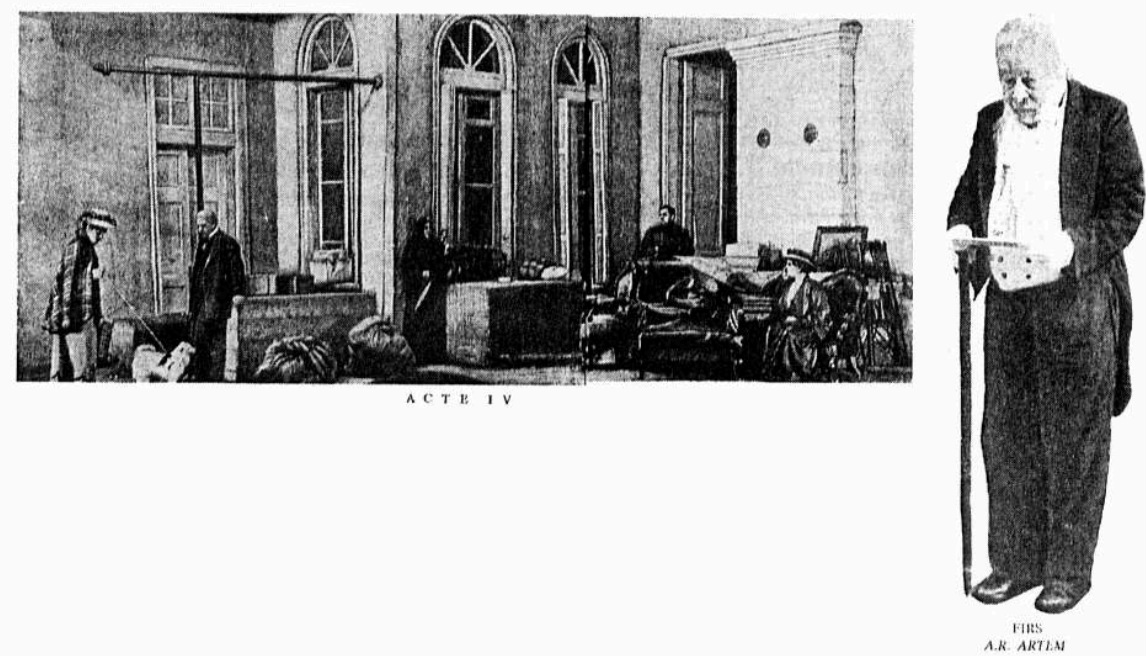

Photo Théâtre d'Art de Moscou

19 J'ai insisté sur l'aisance de Tchékhov dans le genre humoristique, sur les honoraires élevés qu'il pouvait exiger des patrons de presse. Il n'empêche : dès l'origine, le travail proprement littéraire l'occupe, étranger aux auteurs publiant dans les journaux à un sou, il l'occupe à tel point qu'il le distrait de respecter vraiment les règles de la petite presse. Ses papiers sont, au début, quelquefois refusés avec des commentaires très secs. Il aura fallu trois ans pour que Tchékhov se plie aux lois du billet satirique et de 
l'humoresque (1880-1882).

Les petites pièces de Tchékhov, à la différence de ce qu'on trouvait en général dans la presse populaire, sont pleines de références à la littérature russe et étrangère, particulièrement à Dostoïevski et, encore plus, à Gogol. Les citations, les échos de ces deux auteurs parsèment l'ensemble de ses écrits. La maîtrise que Tchékhov avait de la littérature classique, la discrétion et la permanence de l'arrière-fond lyrique, dramatique, romanesque, rendent quasiment impossible le pastiche de ses œuvres. Elles sont beaucoup trop subtiles; trop de voix y résonnent pour qu'on puisse mettre l'accent sur un trait particulier qui, grossi, concentre l'essentiel de son style, et prête à la parodie. «Je suis un gazetier, parce que j'écris beaucoup, mais c'est provisoire. Je ne le resterai pas jusqu'à la fin de mes jours » (1883).

Ayant fait la connaissance de Souvorine, Tchékhov collabore à Temps nouveaux. Sa situation s'améliore. Il gagne plus, et surtout n'est plus pressé de donner des billets satiriques les uns après les autres, sans pouvoir souffler. Il peut publier ses récits sans limitations de longueur, sous son propre nom. A partir de 1887, presque tous les livres de Tchékhov sortent chez Souvorine. Ensemble ils voyagent à travers l'Europe. Souvorine l'aide à recevoir le prix Pouchkine. Tchékhov a, pourrait-on croire, de quoi être un auteur heureux. Mais il garde une grande liberté à l'égard de Souvorine. Il tente de rééquilibrer la ligne du journal, d'en élever le contenu. Avec le temps, il se rend compte que c'est impossible: il y a entre Souvorine et Tchékhov un malentendu de principe. ${ }^{10}$

21 La formation définitive du système esthétique et philosophique de Tchékhov date de 1888 , année de grands changements et de grands succès littéraires. A cette période se précise chez lui une conception de l'œuvre artistique, qui n'a pas grand-chose à voir avec la représentation commune de la littérature comme «école de vie » (manuel de vie, dit-on en russe), courante dans la petite presse, et aussi, malheureusement, dans les revues les mieux considérées. "Vous voulez que, quand je peins des voleurs de chevaux, je dise que c'est mal de voler des chevaux. Ça, c'est une chose qu'on a découverte sans moi il y a belle lurette » (1890). C'est précisément ce que Souvorine ne pouvait admettre. Comme patron de presse, il pensait qu'il fallait répéter tous les jours que le vol est une mauvaise chose. La presse satirique devait dénoncer les détournements de fonds, au jour le jour : il y avait fort à faire. Et c'est en se consacrant à cette tâche que le journal se taillait une clientèle, qu'il se bâtit une réputation. A ce genre de raisonnement Tchékhov répondait placidement: «Les fonctionnaires continuent à recevoir des dessous de table même après avoir lu Gogol ». On comprend mieux le silence de Tchékhov en matière de doctrine.

La vie de la presse suit le modèle suivant: Untel écrit un article, pour qu'un autre le contredise, et qu'un troisième concilie leurs positions. A quoi bon, demande Tchékhov, ce mouvement perpétuel, ce cycle infernal de la critique et de la justification. Le philosophe Lev Chestov en concluait que Tchékhov n'avait pas d'idées, qu'il avait de l'aversion pour les visions du monde. Probablement Chestov se trompait-il à moitié. Il y a bien chez Tchékhov de l'aversion pour le débat d'idées, mais seulement pour celui qui se déroulait dans la presse de son temps. L'atmosphère en était lourde, d'intolérance, de violence polémique. « Vous allez voir, prévient-il en 1888, (...) sous l'étendard de la science, de l'art et de la liberté de pensée menacée, va s'ouvrir chez nous, en Russie, le règne des crapeaux et des crocodiles, comme on n'en a pas vu en Espagne sous l'Inquisition. Vous allez voir! L'étroitesse d'esprit, des prétentions démesurées, un 
amour propre excessif et une complète absence de conscience littéraire et sociale feront leur œuvre. Tous ces Goltsev et $\mathrm{K}^{\circ}$ vont rendre l'air à ce point irrespirable, que tous ceux qui auront gardé quelque fraîcheur d'esprit prendront la littérature en grippe, et que le premier charlatan venu, le premier loup habillé en grand-mère trouvera où se livrer au mensonge, à l'hypocrisie, où mourir " dans l'honneur » ".

La violence dans le débat public en Russie est ancienne. En 1863, le poète Nékrassov, qui dirigeait la revue prestigieuse Le Contemporain conseillait déjà au jeune Leïkine: «Ecrivez, écrivez... Cela vous réussit. Vous connaissez la vie des milieux dont vous parlez. Mais je puis vous donner un conseil... Votre manière est un peu bonasse. Donnez-lui donc, mon cher ami, un peu plus de hargne, de méchanceté... C'est notre époque qui veut ça... Un peu plus de hargne ». On en est resté à cet état d'esprit jusqu'à la fin du siècle, et au-delà.

\section{Tchékhov ou la multiplicité des points de vue}

23 L'objet de la représentation tchékhovienne, ce n'est pas tel ou tel point de vue, telle ou telle position politique, tel programme, juste ou injuste, progressiste ou conservateur, mais la multiplicité des points de vue, fluctuants et contradictoires, qui forment à eux tous ce qui intéressait Tchékhov : la conscience collective au tournant du XXe siècle. L'évidence, dans La Cerisaie comme ailleurs, c'est qu'il est difficile à l'homme de s'élever au-dessus de sa vie particulière, vécue au jour le jour, au-dessus de ses douleurs et de ses soucis, d'accepter la part qui lui échoit.

Pour Souvorine, pour tous les hommes de presse, la vérité est dans les scandales révélés au grand jour, dans l'affirmation sans cesse répétée des valeurs morales les plus universellement admises, qui va naturellement avec la dénonciation des turpitudes des hommes. Eh bien Tchékhov n'est pas sur cette longueur d'ondes. Pour lui la vérité est inconnaissable à l'individu, même au groupe; seule la conscience collective la plus large est capable de l'atteindre. Personne, jamais, n'a raison. Et les gens, au fond, le savent. C'est pourquoi ils sont, dans ses pièces, d'une telle douceur les uns avec les autres, d'une telle indulgence.

Même si Dreyfus est coupable, Zola a raison, car l'affaire des écrivains n'est pas d'accuser, de poursuivre, mais d'intercéder en faveur des coupables, une fois qu'ils ont été condamnés et qu'ils subissent leur peine. On dira: et la politique? et les intérêts de l'Etat? Mais les écrivains, les artistes ne doivent s'occuper de politique que dans la mesure où il faut lui résister. Quant aux accusateurs, aux procureurs, aux gendarmes, ils sont déjà suffisamment nombreux comme ça (...)

Tchékhov affirme que le public est soûlé de haine, rassasié de polémiques. "Notre société vire à l'aigre, comme l'herbe dans un marais ».

On peut voir La Cerisaie à la lumière de cette phrase, qui me tiendra lieu de conclusion : «Les gens ont envie de quelque chose de frais, de libre, de léger, il en ont une envie mortelle»(1900). 


\section{NOTES}

1. Il n'est pas le seul. Voir la réaction de Gorki, citée dans Coulisses, № 14, Mai 1996, page 18.

2. Les intertitres sont de la rédaction

3. Cela n'est pas propre à La Cerisaie. Dans Oncle Vania, au moment où une scène amoureuse semble se nouer, au grand plaisir du spectateur, la séduction, l'érotisme tombent d'un coup : le personnage masculin, Aslrov, déplie une carte d'état-major devant la belle héroïne, et il lui fait tout un exposé sur la destruction des forêts et sur ce qu'il faudrait faire pour améliorer la situation. Que fait le personnage féminin, pendant ce long monologue? Rien : elle l'écoute. Des gestes ténus, des expressions discrètes du visage sont à trouver, pour que la jeune première ne reste pas plantée là pendant le monologue d'Astrov, qui fait soixante lignes.

4. Sur l'importance de la pratique médicale dans la vie de Tchékhov, voir ici même, un peu plus bas.

5. On peut comprendre la perplexité de Stanislavski, si attentif lui-même à relier le texte, le jeu des acteurs, à tout un monde d'objets, devant l'insatisfaction de Tchékhov à l'issue de la première de La Cerisaie. Les fleurs des cerisiers sont «trop grosses ». Les objets sont trop nombreux, trop réalistes, trop pesants : l'état d'âme finit par se perdre. L'univers de la pièce est atomisé en une série d'éléments qui tendent à prendre chacun une signification autonome. Voilà, d'après V. Meyerhold, le grief de Tchékhov à Stanislavski (voir Coulisses, № 14, Mai 1996, page 24).

6. Voir Coulisses, $\mathrm{N}^{\circ} 14$, Mai 1996, page 10.

7. Gorki écrit à Tchékhov : «Vous êtes, je crois, le premier homme libre que j'aie vu, le premier qui ne s'incline devant rien ».

Ce point est important. Les héros de Tchékhov n'ont pas perdu le sens du bien. Le personnage du salaud ne l'intéresse pas particulièrement.

8. Ce point est important. Les héros de Tchékhov n'ont pas perdu le sens du bien. Le personnage du salaud ne l'intéresse pas particulièrement.

9. Voir Coulisses, $\mathrm{N}^{\circ}$ 14, mai 1996, pages 10-11.

10. La rupture se fait à l'occasion des positions très conservatrices prises par le journal sur l'affaire Dreyfus (1899), mais il y a d'autres raisons, moins circonstancielles.

\section{AUTEURS}

\section{SERGE ROLET}

Maître de conférences à l'Université de Franche-Comté 